\title{
Fusiform aneurysm of the basilar artery associated with internal carotid artery agenesis
}

\begin{abstract}
Internal carotid artery (ICA) agenesis is rare and corresponds to the concomitant absence of ICA and the carotid canal. Two possible mechanisms involve the association between ICA agenesis and intracranial aneurysms: hemodynamic overload and hyperflow by demand of a given territory. We describe a case of Type I agenesis of the left ICA associated with fusiform aneurysm and fenestration of the basilar artery (BA).
\end{abstract}

Keywords: internal carotid artery agenesis, flow-related aneurysm, arterial fenestration
Volume I0 Issue 4 - 2020

\author{
Guilherme de Palma Abrão,' Danilo Araujo,' \\ Matheus Miranda Barbosa, ${ }^{2}$ André Didier \\ Lyra,' Alair Augusto Sarmet Moreira Dantas \\ dos Santos' \\ 'Department of Radiology, Fluminense Federal University, Brazil \\ 2Department of Interventional Neuroradiology Rede D’Or \\ Hospitais, Brazil
}

Correspondence: Guilherme de Palma Abrão, Department of Radiology, Fluminense Federal University, Rio de Janeiro, Brazil, Tel +55 21 3741-1662, Email guiabrao@yahoo.com.br

Received: June 16, 2020 | Published: July 17, 2020

\section{Introduction}

Agenesis of the internal carotid artery (ICA) is rare, with an incidence of $0.01 \%$ in the general population. ${ }^{1,2}$ Agenesis on the left is more common than on the right with a $3: 1$ ratio. ${ }^{1}$ The term agenesis represents the complete absence of ICA, with no corresponding carotid canal since its formation follows the development of the artery. The hypoplasia corresponds to the incomplete development of the vessel. ${ }^{3}$ Patients with ICA agenesis are generally asymptomatic but may have a headache, seizures, or neurological deficits secondary to cerebral ischemia or intracranial hemorrhage..$^{1,3}$ Other related symptoms include pulsatile, migraine tinnitus, and Horner syndrome.,

Although an exact cause for these anomalies has not been established, it is known to result from an insult during embryogenesis. The ICA originates from the dorsal aorta and the third aortic arch during the first 6 weeks of embryonic development. On the other hand, the origin of the common and external carotid arteries is controversial. Some authors claim that the ICA and external carotid artery (ECA) originate from the third aortic arch and therefore the agenesis of the ICA should be accompanied by the absence of ipsilateral ECA. For others, common and external carotid arteries can develop normally even in the agenesis of ICA. ${ }^{4}$

Three types of collateral circulation can be observed in the agenesis of ICA: due to the persistence of embryological anastomoses, transdural anastomoses with the ECA or through the Willis polygon. ${ }^{1,3}$ Three types of collateral pathways are described through the Willis polygon: in Type I, the anterior cerebral artery is supplied by the contralateral ACI via the anterior communicating artery (ACoA) and the ACM by the basilar artery via the posterior cerebral artery or ACoP; in Type II, the anterior cerebral arteries and ipsilateral MCA are supplied by the contralateral ACI via ACoA; and in Type III, the anterior cerebral arteries and MCA are supplied by anastomoses that developed from the ACE or contralateral ACI. ${ }^{2}$ Computed tomography (CT) confirms the diagnosis by the absence of the carotid canal. The absence of ICA can be seen in the CT angiographic study. Differential diagnoses include arterial dissection, stenosis, or ICA occlusion. Digital cerebral angiography, on the other hand, can be indicated to assess extra-intracranial collateral circulation and in the association of cerebral aneurysms or other vascular anomalies. ${ }^{6}$

\section{Case report}

A 57-year-old male patient, asymptomatic, with a history of a car accident, in the investigation of head trauma. CT and Angio-CT show the absence of the left carotid canal and non-opacification of the left ICA (Figure $1 \mathrm{~A} \&$ 1B). Other findings are the fenestration and fusiform aneurysm of the basilar artery (BA) with a prominent left posterior communicating artery $(\mathrm{PCoA})$ continuing in the middle cerebral artery (MCA) (Figure $2 \mathrm{~A} \& \mathrm{2B}$ ). Digital cerebral angiography confirmed the findings described and demonstrated that the left middle cerebral artery was supplied by BA through the ipsilateral posterior communicating artery (Figure $3 \mathrm{~A} \& 3 \mathrm{~B}$ ). We consider the demand for flow in the territory of the left MCA by the Type I agenesis of the ICA as a pathophysiological mechanism related to the development of the fusiform aneurysm of BA. Option for conservative treatment of the flow-related aneurysm.

\section{Discussion}

The incidence of intracranial aneurysm in the general population is estimated at 2-4\%, and its association with ICA agenesis occurs in 25$43 \%$ of cases. ${ }^{1}$ Two mechanisms have been postulated to explain the association between ICA agenesis and intracranial aneurysms, may develop secondary to hemodynamic imbalance, or due to an error in embryological development. ${ }^{1}$ The first mechanism is attributed to hemodynamic overload in normal vessels ${ }^{1,7}$ and the higher incidence of these cerebral aneurysms were found in patients over 30 years of age, suggesting that hyperflow-acquired aneurysms are more common than congenital aneurysms. ${ }^{5}$

Fenestration or partial duplication is defined as a division of the vessel lumen, resulting in two distinct endothelium channels that may or may not share the adventitial layer. Arterial fenestrations are described in association with several vascular anomalies, with a strong association between fenestrations and aneurysms, which may originate from these or occur in a different site. ${ }^{8}$ 


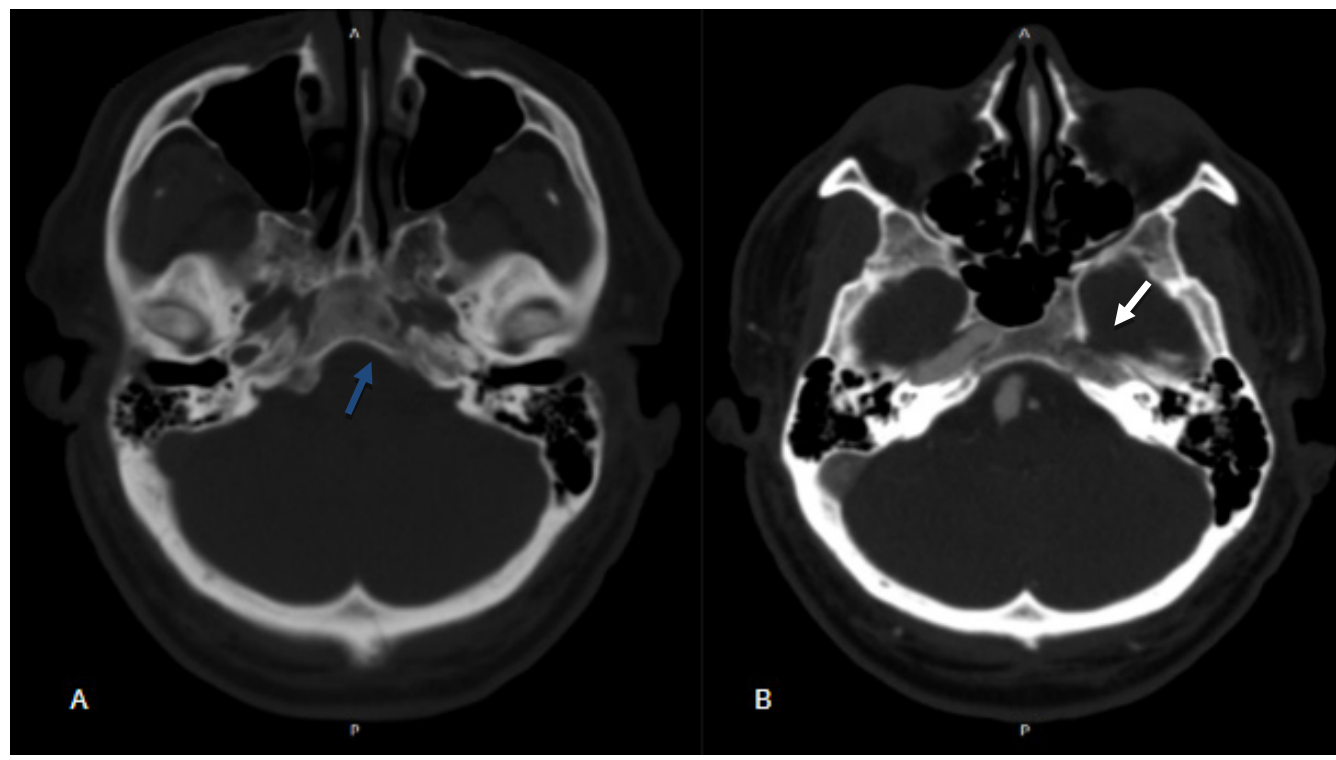

Figure I (A) CT without contrast showing absence of the left carotid canal (blue arrow). (B)-Angio-CT showing non-opacification of the left internal carotid artery (ICA) and absence of the ipsilateral carotid canal (white arrow).

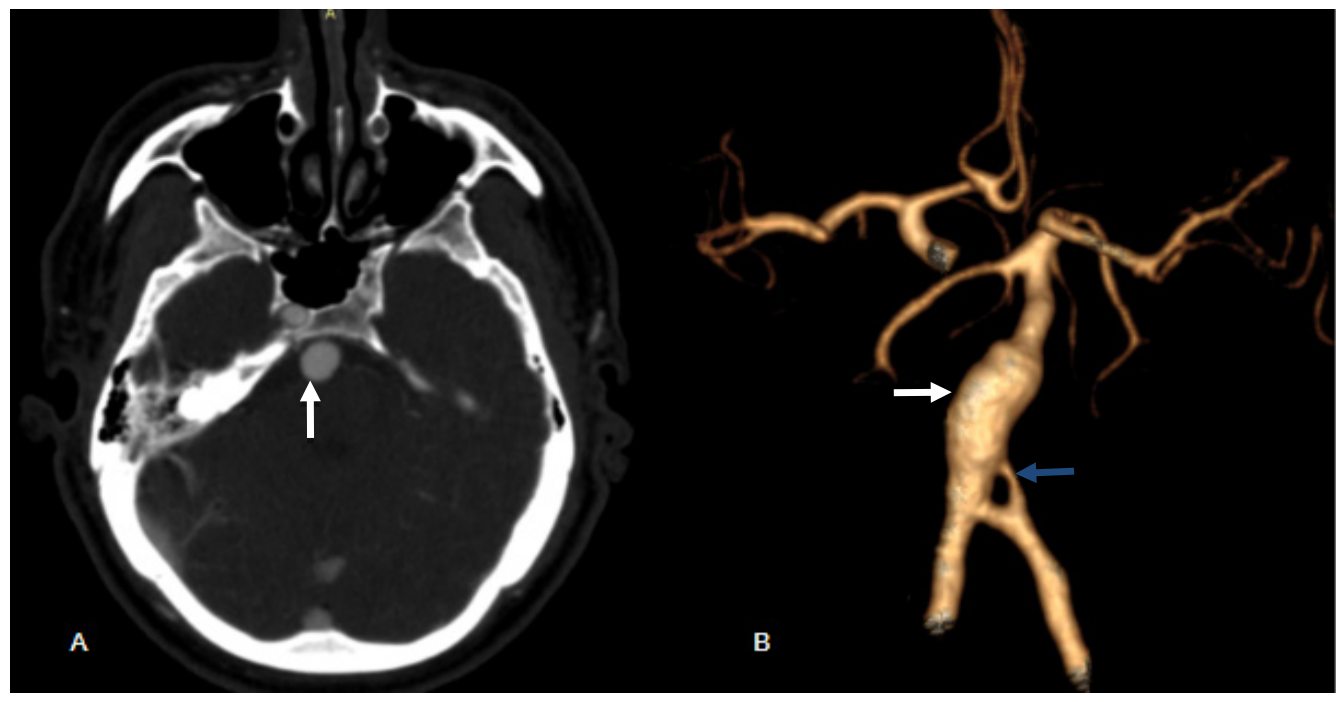

Figure 2 (A) CT angio showing non-thrombosed aneurysm of the basilar artery. (B) Volumetric reconstruction of Angio-CT showing vertebro-basilar fenestration and fusiform aneurysm of the basilar artery (blue arrow).

\section{Conclusion}

In our case, we opted for conservative treatment because it is an asymptomatic patient, considering that the mechanism of hemodynamic overload in the posterior circulation related to ICA agenesis (Type I) ${ }^{2}$ is similar to that related to the development of proximal flow aneurysms in malformations high output cerebral arteriovenous arteries. In these cases, these aneurysmal dilations are usually asymptomatic and do not require an approach. ${ }^{9}$

\section{Acknowledgments}

None.

\section{Conflicts of interest}

The author declares no conflicts of interest.

\section{References}

1. Paşaoğlu L, Vural M, Zıraman I, et al. Left Internal Carotid Artery Agenesis Associated with Basilar and Left Vertebral Artery Aneurysm. J Clin Imaging Sci. 2011;1:60

2. Patel SB, Hashmi ZA, Smaroff GG, et al. Congenital absence of the left internal carotid artery. Ann Vasc Surg. 2010;24(3):415.e9-e11.

3. Lee JH, Oh CW, Lee SH, et al. Aplasia of the internal carotid artery. Acta Neurochir. 2003;145:117-125.

4. Given 2nd CA, Huang-Hellinger F, Baker MD, et al. Congenital Absence of the Internal Carotid Artery: Case Reports and Review of the Collateral Circulation. AJNR Am J Neuroradiol. 2001;22(10):19531959 .

5. Li S, Hooda K, Gupta N, et al. Internal carotid artery agenesis: A case report and review of literature. Neuroradiol J. 2017;30(2):186-191. 
6. Orakdöğen M, Berkman Z, Erşahin M, et al. Agenesis of the left internal carotid artery associated with anterior communicating artery aneurysm: case report. Turk Neurosurg. 2007;17(4):273-236.

7. Elazab NE, Riel-Romero RMS, Gonzalez-Toledo E. Internal carotid artery agenesis and basilar artery aneurysm with third nerve palsy. Pediatr Neurol. 2010;42(6):451-454.
8. Kumar S, Justin EM, Mishra NK. Fenestrated posterior inferior cerebellar artery with concomitant vertebro-basilar junction fenestration and vertebral artery aneurysm. Clin Neuroradiol. 2012;22(3):235-237.

9. Xianli LV, Zhongxue WU, Hongwei HE, et al. Proposal of Classification of Aneurysms Coexisting with AVM and Possible Treatment Strategies. Turk Neurosurg. 2016;26(2):229-233. 